\title{
Indian Music Reorganizes Time and Establishes Homeostasis
}

\section{P.Barathi *}

\section{HOMEOSTATIC EFFECTS OF RAG AHIR BHAIRAV ON THE CIRCULATORY SYSTEM \\ “ Music can form a bridge between external and internal worlds making whole out of apparent disparate elements"}

According to Mozart, man is an antenna, a ceaseless receptor of the waves and rhythms in the universe( Alfred Tomatis, 1991). The biological and neuro-physiological rhythms are attuned to and in balance with the cosmic rhythms that are beyond human auditory perception. Music awakens the fundamental rhythms existing in each of us. Cardiac and respiratory rhythms are made to synchronize with the deeper, innate rhythms existing in each of us through the medium of music putting man into resonance with the universe. Indian classical ragas can adapt the eternal rhythms to our neurons, and the body in turn resonates in tune with the melodic modes. The latent harmony existing in each of us is aroused, and the body re-establishes its lost equilibrium.

The homeostasis established by music administration has been documented in a study conducted in Tokai University, Japan. The study reveals that music has homeostatic effects on emotion, when listeners were analysed by the Profile of Mood States (POMS), a psychological test for measuring emotion (yamamoto et al., 1999).

\section{HOW DOES INDIAN MUSIC ESTABLISH HOMEOSTASIS?}

Music exerts homeostatic effects by harmonizing psychological and biological time. Music, as an external oscillator entraining our internal oscillators, has the potential to affect not only our sense of time, but also our sense of being in the world ( Mari Reiss jones,1989). Jones postulates a propensity for an individual's endogenous rhythms to synchronize with the musical item, the entrainment experiences varying according to physiological, and psychological factors, as well as the stage of development of the listeners exposed to identical musical pieces. The subjects slowly drift into the realm of 'reorganized time', the metaphysical domain where intellectual abilities are replaced by imaginary realities.

I have documented several case studies after administering Rag Ahir Bhairav to patients with acute as well as chronic hypertension, and was amazed to find that it not only brought down the elevated blood-pressure in hypertensives but also restored the normal blood pressure in hypotensive individuals. An exploration into the entrainment properties of music will throw a light on such homeostatic phenomena.

\section{Brain, Entrainment, ANd Physiological Rhythms}

Two aspects of the new view on the brain seem to be relevant in this context : a) most brain functions can best be described as cooperative, synchronized activity of large,distributed ensembles of neurons, and b) a large part of this synchronized activity is of an oscillatory nature(Basar 1983; Nunez et al 1993). These autorhythmic oscillatory properties of neurons in the central nervous system are a consequence of their electrochemical properties. The cooperative and oscillatory activities of these neurons can be seen, amongst others, as the basis for the timing of sensory-motor coordination ( Llinas 1988). With these new views on the functioning of the brain, it seems most promising to apply the concept of entrainment to the analysis of human interactions at the interpersonal and social level as well. Indeed, such applications have been pursued in the social sciences in parallel with the development of neuroscientific approaches, the mathematics of coupled oscillators, and numerous other related strands of research(Martin Clayton et al,2004)..

A good deal of current medical research is concerned with the behaviour of endogenous physiological rhythms in humans (such as the variation of body temperature over the 24-hour cycle), and how the study of those rhythms might be further developed as a tool in the diagnosis of pathological states and ultimately lead to the development of

\footnotetext{
* Dr. P.Barathi, M.D.,

Prof of Physiology, Music Therapist

e-mail : iyer.bharthi@gmail.com
} 
new treatments ( Glass 1996). An important part of this work is the consideration of entrainment and in particular, identifying which physiological rhythms entrain to which stimuli, and under what conditions. There are many examples where relatively stable and entrained biological rhythms are associated with good health (the enhanced stability of the heart rate afforded by a pacemaker is an example of this), while conversely asynchrony and instability of rhythmic processes can be associated with pathologies.

The behaviour of our endogenous cardiac rhythm ought to be predictable when stimulated by a pacemaker, but if the period of the pacemaker were set outside a certain range, the behaviour of the cardiac rhythm would be for practical purposes unpredictable. But entrainment does not necessarily imply stability of biological rhythms, and in any case stability is not necessarily associated with good health. In the case of brain waves, for example, we have a different pairing: stable brain waves may indicate a pathology (epilepsy) while unstable waves may indicate a healthy state. Indeed, a certain amount of flexibility and dynamic equilibrium seems to be associated with health in many systems.

Examples of endogenous or naturally occurring rhythms within the human body include the heartbeat, blood circulation, respiration, locomotion, eyes blinking, secretion of hormones, female menstrual cycles, and many others. It has been suggested, indeed, that all human movements are inherently rhythmic: Jones writes that "All human performance can be evaluated within a rhythmic framework".

Music psychologists have begun to apply an entrainment model in which rhythmic processes endogenous to the listener entrain to cues in the musical sound (Large and Kolen 1994). Although there is much to be done in this area, the entrainment model seems to reflect the cognitive processes much better than do previous other models.

\section{WHAT IS SPECIAL ABOUT MUSICAL ENTRAINMENT?}

It seems that certain kinds of musicking can afford particularly precise synchronisation between individuals' behaviours. This may in fact be one of the basic reasons for the development of different kinds of musical repertories, since musical metre is often (although is not invariably) more regular, with more hierarchical levels, than the rhythmic patterning of speech and other communicative behaviour. Since certain degrees of entrainment between individuals seem to be associated with positive affect, it may be the case that particular patterns, periodicities, hierarchies or intensities of entrainment afford particular affects. It is also possible that particular kinds of music may promote the switching of attention between temporal levels.

Musical experience, while individually unique in every case, is nevertheless always social. Through exploring the phenomena of entrainment, ethnomusicologists are able to better understand how musical sound serves as an interface that connects selves—viscerally and cognitively-to society.

Measured music is a realm of 'reorganized time', but so is non-measured music. Both types of music display high temporal coherence, at least if they are studied as dynamic systems, not as static products. A brief glance at Chinese folk songs may serve to illustrate this. There are several Chinesefolk song performances in which the lyrics sung are essentially meaningless. In these performances the whole act of singing seems meaningless, except in its quality of 'organized time': a capacity of people to employ sound (i.e. voices) to revive bonds and fates shared beyond daily realities, in the realm of metaphysics.

Just how many new hard core facts we will be able to unearth via 'entrainment' remains to be seen: if entrainment is the study of 'reorganized time', it is also, by definition,the domain of metaphysics - a realm where poets operate more successfully than most academics.

Indeed, "rhythmical entrainment" and its physicality are not everything. The bio-physicism of rhythmical entrainment leads to the attuning of other psychological realms, to the transformation of persons' state of mind, which is also a form of periodicity. By disconnecting the mind from its ordinary cycles (regularities or irregularities), inner oscillators and oscillations set up an affected perception, sensitivity or emotional availability. This is how - in an "extended" version of the theory of entrainment - the contextual variety of music's effects can be understood or explained.

Physical entrainment refers to the synchronization of concrete objects in the world, determined by some objective measure, while cognitive entrainment refers to the appearance of synchronization as determined by the subjective 
measure of an observer or participant. Cognitiveen trainment is the process whereby an individual comes to sense a certain degree of synchronization between two or more rhythmic processes in the world.

From the above references, we can hypothesize that some heptatonic ( having all the 7 notes of the octave) ragas like Ahir bhairav have the inherent capacity to 'cognitively reorganize' psychological time, thereby influencing the biological time and the associated homeostatic mechanisms, rectifying the disordered homeostasic mechanisms towards their normal rhythmic balance.

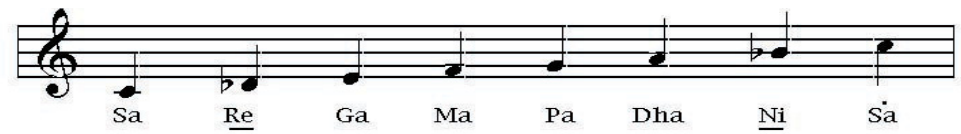

About Rag Ahir Bhairav

It is characterized by the use of Komal Re and Komal Ni. Often times, this rag is referred to by its south Indian

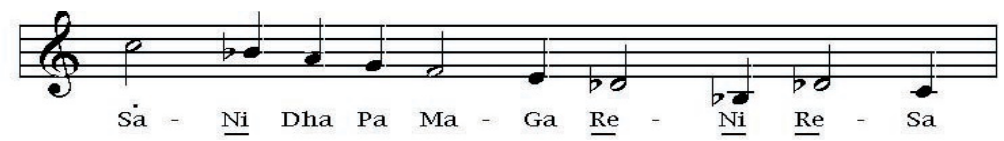

name

of Chakravaka. This is an early morning rag. .

Arohana

Avarohana

Jati :Sampurna - Sampurna;Time-Early Morning :That-Chakravaka

\section{REFERENCES}

1. Alfred Tomatis(1991).”Pourquoi Mozart ?” Paris: first edition, pp-91-92.

2.. Basar, E. (1983) EEG and synergetics of neural populations. In Basar, E. et al. (Eds.), Synergetics of the brain (pp. 183-200). Berlin, Heidelberg, New York: Springer.

3. Clayton, M., Sager, R., and Will, U. (2004). In time with the music: The concept of entrainment and its significance for ethnomusicology. ESEM CounterPoint, 1, 1-45.

4. Glass, L. (1996). Synchronization and rhythmic processes in physiology. Nature, 410 (6825), 277-284.

5. Jones, M.R. (1976). Time, Our Lost Dimension:Toward a New Theory of Perception, Attention, and Memory. Psychological Review, 83(5), 323-355.

6 . Large, E.W. and Kolen, J.F. (1994). Resonance and theperception of musical meter. Connection Science: Journal of Neural Computing, Artificial Intelligence and Cognitive Research, 6(2-3), 177-208.

7 . Llinas, R. (1988). The intrinsic Electrophysiological Properties of Mammalian Neurons. Science, 242,1654-1664.

8. Nuñez, A., Amzica, F., and Steriade, M. (1993).Electrophysiology of Cat Association Cortical Cells in Vivo: Intrinsic Properties and Synaptic responses. Journal of Neurophysiology, 70, 418-430.

9. Yamamato Kenji, Iga Tomiei, Takahashi Sachiko, Schimuzu Tetsuo (1999). Tokai journal of Sports Medical science: Tokai universitybehavior, for example, continuing the music if the subject attentively listens to it, and vice versa. The 